\title{
Unidirectional water-transport antibacterial trilayered nanofiber-based wound dressings induced by hydrophilic- hydrophobic gradient and self-pumping effects DOI:
}

10.1016/j.matdes.2021.109461

\section{Document Version}

Final published version

Link to publication record in Manchester Research Explorer

Citation for published version (APA):

Qi, L., Ou, K., Hou, Y., YUan, P., Yu, W., Li, X., Wang, B., He, J., Cui, S., \& Chen, X. (2021). Unidirectional watertransport antibacterial trilayered nanofiber-based wound dressings induced by hydrophilic-hydrophobic gradient and self-pumping effects. Materials \& Design, [109461]. https://doi.org/10.1016/j.matdes.2021.109461

\section{Published in:}

Materials \& Design

\section{Citing this paper}

Please note that where the full-text provided on Manchester Research Explorer is the Author Accepted Manuscript or Proof version this may differ from the final Published version. If citing, it is advised that you check and use the publisher's definitive version.

\section{General rights}

Copyright and moral rights for the publications made accessible in the Research Explorer are retained by the authors and/or other copyright owners and it is a condition of accessing publications that users recognise and abide by the legal requirements associated with these rights.

\section{Takedown policy}

If you believe that this document breaches copyright please refer to the University of Manchester's Takedown Procedures [http://man.ac.uk/04Y6Bo] or contact uml.scholarlycommunications@manchester.ac.uk providing relevant details, so we can investigate your claim.

\section{OPEN ACCESS}




\title{
Unidirectional water-transport antibacterial trilayered nanofiber-based wound dressings induced by hydrophilic-hydrophobic gradient and self-pumping effects
}

\author{
Linya $\mathrm{Qi}^{\mathrm{a}}$, Kangkang Ou ${ }^{\mathrm{a}, \mathrm{b}, *}$, Yijun Hou ${ }^{\mathrm{a}}$, Pingping Yuan ${ }^{\mathrm{a}}$, Wen $\mathrm{Yu}^{\mathrm{a}}$, Xiang Li ${ }^{\mathrm{a}}$, Baoxiu Wang ${ }^{\mathrm{b}}$, \\ Jianxin He ${ }^{\mathrm{a}, \mathrm{c}, *}$, Shizhong Cui ${ }^{\mathrm{a}, \mathrm{c}}$, Xiaogang Chen ${ }^{\mathrm{a}, \mathrm{d}}$ \\ a Textile and Garment Industry of Research Institute, Zhongyuan University of Technology, Zhengzhou 450007, PR China \\ b State Key Laboratory for Modification of Chemical Fibers and Polymer Materials, College of Materials Science and Engineering, Donghua University, Shanghai 201620, PR China \\ c Collaborative Innovation Center of Textile and Garment Industry, Zhengzhou 450007, Henan, PR China \\ d The University of Manchester, Manchester M13 9PL, UK
}

\section{H I G H L I G H T S}

- The unidirectional water-transport wound dressing with hydrophobichydrophilic gradient and self-pumping effect are designed.

- The dressing can effectively drive the wound exudates outflow and prevent those pumped biofluids rewetting the wounds.

- The dressing with PHGC has excellent antibacterial and low cell-adhesion effect, showing potential application in wound care.

\section{A R T I C L E I N F O}

\section{Article history:}

Received 30 November 2020

Received in revised form 5 January 2021

Accepted 7 January 2021

Available online 11 January 2021

\section{Keywords:}

Wound dressing

Multilayer nanofibrous membrane

Unidirectional water-transport

Hydrophilic-hydrophobic gradient

Self-pumping effect

\section{GRA P H I C A L A B S T R A C T}

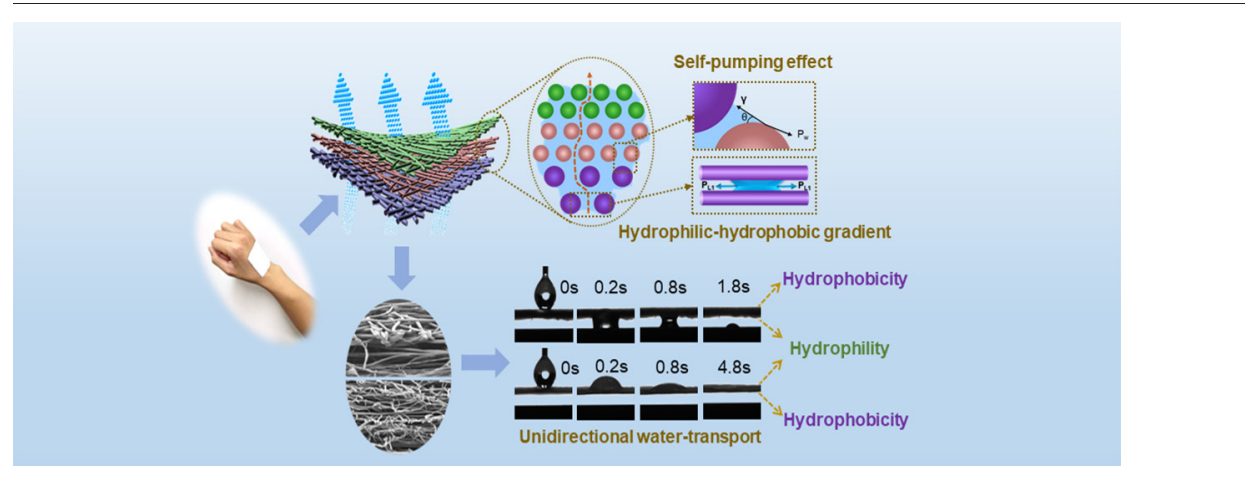

\begin{abstract}
A B S T R A C T
Excessive wound exudates often cause infection and hinder wound repair and regeneration. It is therefore urgently needed to develop an ideal wound dressing for pumping the excessive biofluids and inhibiting bacterial infection in the wound healing process. Herein, we demonstrate a facile and efficient strategy to fabricate the unidirectional water-transport antibacterial wound dressings by embedding macromolecular antimicrobials into a multilayer nanofibrous membrane featured with hydrophobic-to-hydrophilic gradient structure and selfpumping effect using an electrospinning technique. Thanks to these features, the obtained nanofibrous dressings achieved excellent unidirectional water-transport performance, which could drive wound exudates to flow spontaneously from inside to outside and prevent pumped biofluids rewetting the wounds. In addition, the designed trilayered dressing exhibited approximately $100 \%$ antibacterial ability against Staphylococcus aureus and Escherichia coli when the concentration of antibacterial agents polyhexamethylene guanidine hydrochloride (PHGC) was $0.06 \mathrm{wt} \%$. Moreover, the trilayered dressings revealed excellent water absorption performance, air and moisture permeability, mechanical strength, biocompatibility, and low cell adhesion behavior, indicating the potential applications for the trilayered nanofibrous wound dressings in wound care.
\end{abstract}

(c) 2021 Published by Elsevier Ltd. This is an open access article under the CC BY-NC-ND license (http:// creativecommons.org/licenses/by-nc-nd/4.0/).

* Corresponding authors at: Textile and Garment Industry of Research Institute,

Zhongyuan University of Technology, Zhengzhou 450007, PR China.

E-mail addresses: kkou@zut.edu.cn (K. Ou), hejianxin771117@163.com (J. He). 


\section{Introduction}

The integrity of human skin plays a critical role in maintaining the osmotic balance of the body. When the skin is injured, bleeding emerges and causes exudates, which bring considerable challenges to wound care. Biofluid management of wounds is an essential precondition for wound repair, involving the removal of excessive biofluid [1]. Therefore, it is necessary to manage the biofluid at the wound site with an appropriate material. Moisture dressings are currently the most common method for wound treatment and can provide a favorable microenvironment to accelerate the healing process [2,3]. Although conventional moisture dressings can maintain a moist microenvironment at the wound site, excessive exudates overhydrate the wounds, which often causes infection and hinders wound healing [1]. Accordingly, it is essential to develop a unidirectional water-transport antibacterial wound dressing to drain excessive biofluid from wounds and maintain a suitable microenvironment to accelerate wound healing.

To date, unidirectional water-transport fabrics have been fabricated primarily by constructing a hydrophilic-hydrophobic asymmetric bilayer structure with a surface energy gradient in the thickness direction, resulting in an additional pressure difference from the hydrophobic region to the hydrophilic region to achieve unidirectional water-transport effect [4-8]. Recently, various kinds of techniques have been developed to fabricate the unidirectional water-transport fabrics with hydrophilichydrophobic gradient structure, including the chemical finishing [9], surface modification [10], in situ polymerization [11]. Nonetheless, most of these strategies involved a complicated fabrication process or the prepared unidirectional water-transport fabrics exhibited poor durability $[12,13]$. It is therefore urgently needed to develop a more feasible and effective strategy to fabricate the unidirectional water-transport wound dressing with the reliable structural designability $[14,15]$.

Notably, electrospinning technique has been proven to be an efficient, scalable, and versatile technique to fabricate highly interconnected nanofibrous mats. Due to their unique merits, such as a large specific surface area, good breathability, high mechanical flexibility, easily deformable porous structure and extracellular matrix-biomimetic structure, electrospun nanofibrous mats have shown significant advantages in the construction of delicate structures for wound care [16-18]. Based on these advantages, we attempted to construct trilayered nanofibrous unidirectional water-transport wound dressings by employing the inherent hydrophobic-to-hydrophilic properties of polyurethane (PU), polyacrylonitrile (PAN), and sodium polyacrylate (SPA) [19-22]. In more detail, the hydrophobic PU nanofibrous membrane was served as innermost layer to contact with the wound, in order to reduce adhesion between dressing and wound and prevent the exudates from rewetting the wounds. The hydrophilic PAN-SPA nanofibrous membrane was served as outermost layer to contact with air, abundant micropores formed by interlaced nanofibers can effectively isolate external bacteria. The intermediate hydrophilic PU/PAN-SPA nanofibrous membrane is designed as the drainage layer, which can absorb and pump the exudates efficiently. Additionally, the PU component with excellent mechanical properties of the trilayered nanofibrous membrane enable the stable gradient structure for the wound dressing $[23,24]$.

The moist microenvironment is beneficial for promoting the wound healing process; meanwhile, bacteria thrive in the moist microenvironment. The presence of bacteria can cause wound infection and inflammation, and in severe cases, can be life-threatening [25,26]. Traditional dressings often use small-molecule antibacterial agents, which have the advantage of rapid sterilization, but they can also enter the human body and affect human health $[27,28]$. Although nano-silver exhibits an excellent antibacterial effect, studies have demonstrated that its complete safety is still to be achieved [29,30]. Polymer antibacterial agents with high safety and long-term antibacterial properties have attracted considerable interest in the antibacterial field [31,32]. The guanidine group-containing cationic polymer antibacterial, similar to the structure of natural antibacterial peptides, has outstanding antibacterial properties and excellent biocompatibility. Constructing it into wound dressings can effectively prevent the bacterium from breeding at the wound surfaces [33-35].

In summary, we design a unidirectional biofluid transport antibacterial trilayered nanofiber-based wound dressings by incorporating polymer antibacterial agents into multilayer nanofibers featured with hydrophobic-to-hydrophilic gradient structure and self-pumping effect by utilizing the electrospinning technique. The resultant gradient structure can drive wound exudates to flow spontaneously from inside to outside and achieve unidirectional water-transport effect. Furthermore, the trilayered dressing exhibited excellent antibacterial effect when the concentration of antibacterial agents PHGC was $0.06 \mathrm{wt} \%$. In addition, the dynamic contact angle of the trilayered dressing with a gradient structure decreased from $133^{\circ}$ to $0^{\circ}$ in $2 \mathrm{~s}$. The equilibrium water content, water absorption, breaking strength, air permeability, and water vapor transmission rate of the multilayer dressing were $95 \%, 1323.6 \%$, $6.7 \mathrm{MPa}, 6.8 \mathrm{~mm} / \mathrm{s}$, and $1360 \mathrm{~g} / \mathrm{m}^{2} /$ day, respectively, which meet the requirements of the ideal wound dressing.

\section{Materials and methods}

\subsection{Materials}

PU was purchased from BASF (Germany). PAN with an average molecular weight of $7.0 \times 10^{5}$ was purchased from Suzhou Huihuang Fluoroplasticization Co., Ltd. (China). SPA was obtained from InBev Biotechnology Co., Ltd. (Hangzhou, China). N, N-dimethylformamide (DMF), tetrahydrofuran (THF), and ethanol (EtOH) were purchased from Tianjin Fuyu Fine Chemical Co., Ltd. (China). Guanidine hydrochloride and 1, 6-hexylenediamine were purchased from Sinopec Chemical Reagent Co., Ltd. (China) and used without further purification. Human gastric mucosal epithelial cells (GES-1) were purchased from Shanghai Institute of Biochemistry and Cell Biology, Chinese Academy of Sciences. MTT reagent, Dulbecco minimum essential medium (DMEM) and fetal bovine serum (FBS) were purchased from Thermo Fisher Scientific Inc.

\subsection{Preparation of trilayered wound dressing}

The fabrication of trilayered nanofibrous dressings is as follows: A PU solution of $18 \mathrm{wt} \%$ in DMF/THF (mass ratio = 5:5) was prepared at room temperature with constant stirring for $6 \mathrm{~h}$. A mixed solution of $12 \mathrm{wt} \%$ PU/PAN (mass ratio $=7: 3$ ) in DMF was prepared and stirred continuously for $6 \mathrm{~h}$ at room temperature. Next, SPA was added with a relative PAN of $0,2,4,6,8,10$, and $12 \mathrm{wt} \%$ and stirred evenly to obtain the final PAN/PU-SPAx spinning solution. A PAN solution of $12 \mathrm{wt} \%$ PAN in DMF was obtained, and then SPA with a relative PAN of $10 \mathrm{wt} \%$ was added and stirred evenly to obtain the PAN-SPA10 spinning solution. The trilayered nanofibrous dressings were prepared by electrospinning. The multilayer membranes from inside to outside are in turn: PU, PU/ PAN-SPA and PAN-SPA membrane. The as-prepared spinning solution was transferred to $10 \mathrm{~mL}$ syringes with blunt metal needles of $0.41 \mathrm{~mm}$ inner diameter that was fed at a constant rate of $0.1 \mathrm{~mL} / \mathrm{min}$. The spinning distance, voltage, temperature, and humidity were controlled at $18 \mathrm{~cm}, 20 \mathrm{kV}, 25 \pm 5{ }^{\circ} \mathrm{C}$ and $35 \pm 5 \%$, respectively. The electrospinning time of the PU fibrous membrane was set as 1,2, and $3 \mathrm{~h}$. The thicknesses of PAN/PU-SPA and PAN-SPA nanofibrous membranes were controlled at $150 \mu \mathrm{m}$.

\subsection{Fabrications of PHGC and antibacterial nanofibrous dressing}

The synthetic of PHGC is shown as below [36]. 48 g guanidine hydrochloride and $58 \mathrm{~g} \mathrm{1,6-hexylenediamine} \mathrm{were} \mathrm{placed} \mathrm{in} \mathrm{a} \mathrm{three-necked}$ flask at $120^{\circ} \mathrm{C}$ for $2 \mathrm{~h}$. The reaction temperature continues to rise to $180{ }^{\circ} \mathrm{C}$ after the $\mathrm{NH}_{3}$ was completely released. Next, the mixture was stirred for $6 \mathrm{~h}$, and the final product was collected after drying under $100{ }^{\circ} \mathrm{C}$. PHGC was added to the inner and outer fibrous membranes of 
the wound dressing according to $0,0.02,0.04,0.06$, and $0.08 \mathrm{wt} \%$ of the total polymer amount.

\subsection{Testing and characterization}

\subsubsection{Morphology characterization}

The surface morphology of the nanofiber membranes was characterized using a Zeiss SIGMA-500 field emission scanning electron microscope (FESEM), and the cross-sectional morphology of the wound dressings was observed using a US Phenom scanning electron microscope. From SEM images, the average diameter of the fibers was measured using ImageJ software (National Institutes of Health, USA, $n=100$ ).

\subsubsection{Water contact angle test}

The water contact angle (WCA) of the samples was measured using an optical contact angle tester (Data physics, Germany) with $2 \mu \mathrm{L}$ and $5 \mu \mathrm{L}$ water droplets. The pictures were captured at predetermined times to measure the dynamic contact angle of the water droplets at different positions on the fiber membranes and record the time required by the water droplets to be completely absorbed on different fiber membranes.

\subsubsection{Water absorption performance test}

The water absorption performance of the wound dressing directly affects the wettability of the wound, and the moist environment is beneficial for wound healing. The hydroscopicity and equilibrium water content of the material were characterized to explore whether the hydroscopicity of the prepared nanofiber membranes meets the requirements of wound dressings. First, a $20 \times 20 \mathrm{~mm}^{2}$ dried sample was immersed in $10 \mathrm{~mL}$ deionized water for $2 \mathrm{~h}$ at $37^{\circ} \mathrm{C}$. Next, the sample was removed and weighed after gentle surface wiping using filter paper. The water absorption and equilibrium swelling ratio were calculated according to Eqs. 1 and 2:

Water absorption $(\%)=\frac{W_{1}-W_{0}}{W_{0}} \times 100 \%$

Equilibrium water content $(\%)=\frac{W_{1}-W_{0}}{W_{1}} \times 100 \%$

where $W_{1}$ and $W_{0}$ are the weights of the absorbed and dried samples, respectively.

\subsubsection{Air permeability test}

Wound dressings should be breathable and moisture permeable to maintain the exchange of gas at the wound [37]. The air permeability of the nanofiber membrane was measured using a YG461E-III automatic air permeability meter. The pressure difference between the internal and external of the nanofiber membrane was controlled at $100 \mathrm{~Pa}$, and the test area was $20 \mathrm{~cm}^{2}$. The tests were randomly conducted at 10 positions of the nanofibrous membrane.

\subsubsection{Water vapor transmission rate test}

The water vapor transmission rate (WVTR) of the nanofibrous membranes was evaluated according to the ASTM E96 testing standard. In brief, a cylindrical cup containing distilled water was covered with the sample and put into a constant temperature and humidity box. The environmental parameter temperature, relative humidity, and airflow were $38{ }^{\circ} \mathrm{C}, 2 \%$, and $0.5 \mathrm{~m} / \mathrm{s}$, respectively. Each sample was tested three times, and the average value was regarded as WVTR of the nanofibrous membrane. The WVTR was calculated according to Eq. 3:
$\operatorname{WVTR}\left(\mathrm{g} / \mathrm{m}^{2} /\right.$ day $)=\frac{G}{1000 \times t \times A} \times 24$

where $G(\mathrm{~g})$ denotes the mass change of the permeable cup in $t$ (h) test time, and $A$ represents the effective test area of the cup mouth $\left(\mathrm{m}^{2}\right)$.

\subsubsection{Mechanical performance test}

The mechanical properties of the nanofiber membranes were tested using an XLW intelligent electronic tensile testing machine. All membranes were cut into five strips of $50 \times 10 \mathrm{~mm}^{2}$, and the thickness of each sample was gauged using digital display thickness numerous times. Each sample was tested five times at a crosshead speed of $10 \mathrm{~mm} / \mathrm{min}$, and the average values of both tensile strength and elongation at break were recorded.

\subsubsection{Droplet diffusion rate test}

The diffusion rate of the droplets was tested using $50 \mu \mathrm{L}$ deionized water with blue ink to investigate the process of water-transport in the nanofibrous membrane. The membranes were placed horizontally on the top and bottom sides during the measurement. The diffusion diameter of droplets in a single-layer fibrous membrane and trilayered wound dressings was recorded every $10 \mathrm{~s}$. The unidirectional watertransport performance of trilayered wound dressings was also measured by the WCA on the top and bottom surfaces of the dressings.

\subsubsection{FTIR spectrum and TG analysis}

PHGC, PAN, and PAN-PHGC samples were dried and ground to powder. FTIR spectra of the samples were measured using the $\mathrm{KBr}$ compression method on a Varian 640-IR Fourier infrared spectrum tester. The absorption mode was selected as the test condition in the wavenumber range from 4000 to $400 \mathrm{~cm}^{-1}$.

The TG curves of the samples were measured using a TG209Fl thermogravimetric analyzer. The test conditions were as follows: the sample mass, temperature range, and heating rate were $5 \mathrm{mg}, 35-800{ }^{\circ} \mathrm{C}$, and $10{ }^{\circ} \mathrm{C} / \mathrm{min}$, respectively, and argon was used as the protective gas under $\mathrm{N}_{2}$ atmosphere.

\subsubsection{Antimicrobial test}

The trilayered antibacterial dressing was prepared by adding PHGC to the inner the outer layer of the spinning solution. The most common gram-negative bacteria (E. coli) and gram-positive bacteria (S. aureus) were used as experimental strains. Wound dressing samples with different PHGC concentrations were cut into discs with a diameter of $2 \mathrm{~cm}$. After high-temperature and high-pressure sterilization, the wound dressing samples were placed into a bacterial solution of the same concentration of culture for $2 \mathrm{~h}$. $0.1 \mathrm{~mL}$ culture solution was taken and evenly coated on the agar medium, and the wound dressing samples were cultured at $37^{\circ} \mathrm{C}$ for $12 \mathrm{~h}$ to observe the antibacterial effect.

PHGC dissolution resistance was analyzed by bacteriostatic circle PHGC solid with $0 \mathrm{wt} \%$ and $0.06 \mathrm{wt} \%$ PHGC concentration of the inner and outer layers of the antimicrobial properties of the nanofiber membrane. The sample was cut into wafers of $2 \mathrm{~cm}$ diameter, after hightemperature and high-pressure sterilization, in the same concentration of bacteria with $0.1 \mathrm{~mL}$ of the agar medium and $37^{\circ} \mathrm{C}$ constant temperature culture for $12 \mathrm{~h}$ to observe the antibacterial effect.

\subsubsection{In vitro cytotoxicity tests}

The in vitro biocompatibility of these membranes was assessed to investigate the viability of GES-1 using MTT assay [2]. Briefly, the $10 \times 10 \mathrm{~mm}^{2}$ of trilayered membranes were extracted using $10 \mathrm{~mL}$ DMEM with $10 \%$ FBS for $24 \mathrm{~h}$ at $37^{\circ} \mathrm{C}$. GES- 1 were cultured in DMEM containing $10 \% \mathrm{FBS}, 100 \mu \mathrm{g} / \mathrm{mL}$ streptomycin and $100 \mathrm{U} / \mathrm{mL}$ penicillin at $37{ }^{\circ} \mathrm{C}$ with $5 \% \mathrm{CO}_{2}$ incubator. Thereafter, GES-1 were seeded and cultured $24 \mathrm{~h}$ in 24 -well plates at $1 \times 10^{4}$ cells/well, then the medium was 
replaced with $100 \mu \mathrm{g}$ extracts from the samples. After $48 \mathrm{~h}$ incubation, the medium was pipetted out and replaced with the fresh medium with MTT solution. After incubating for $4 \mathrm{~h}$ at $37^{\circ} \mathrm{C}$, the absorbance was measured at $490 \mathrm{~nm}$ using an enzyme labeling instrument. All the measurements were performed three parallel experiments. Culture medium without extracts was used as control.

Cell adhesion test was conducted as follows. First, the samples were sterilized in $75 \mathrm{vol} \%$ ethanol vapors for $4 \mathrm{~h}$ and immersed in medium for $2 \mathrm{~h}$. Then, GES-1 were seeded on the PU inner layer and PAN-SPA outer layer of trilayered wound dressings at $1 \times 10^{4}$ cells/well. The culture medium was replenished every day and the cells were cultured for $48 \mathrm{~h}$ at $37{ }^{\circ} \mathrm{C}$ and $95 \% \mathrm{RH}$ with $5 \% \mathrm{CO}_{2}$. After incubating $48 \mathrm{~h}$, the cells cultured on samples were fixed by $4 \%$ paraformaldehyde and dehydrated with graded tertiary butanol (40-100\%) and further lyophilization. Ultimately, the samples were observed by SEM.

\subsubsection{Statistical analysis}

Statistical analysis was conducted using Origin 8 (GraphPad Inc., San Diego, CA). All experimental data were calculated as the mean \pm standard deviation of at least three independent samples, with a significant difference $p<0.05$.

\section{Results and discussion}

\subsection{Fabrication of trilayered nanofiber-based wound dressings}

An ideal wound dressing should possess unidirectional watertransport effect for the purpose of pumping the wound exudates and prevent it from reverse osmosis. Fig. 1a clearly illustrates the fabrication process of the wound dressing, which primarily involves three layers: hydrophobic PU, hydrophilic PU/PAN-SPA, and super-absorbent PANSPA. Briefly, a thin PU nanofibrous layer was spun as the low-adherent inner layer; then, the PU/PAN-SPA layer was spun on the inner layer as a drainage intermediate layer; finally, the PAN-SPA layer was spun as a reservoir outer layer. The trilayered wound dressings with hydrophobic-hydrophilic gradient structure were obtained by combining the inner, middle, and outer layer membranes. To endow the wound dressing with specific antibacterial properties for preventing wound infections, PHGC was synthesized as an antibacterial agent and added to the dressings. The synthetic route of PHGC is demonstrated in Fig. 1d, and the properties of wound dressings will be discussed in more detail later.

\subsection{Morphology of nanofibrous membrane}

The SEM images in Fig. 1c illustrate that PU, PU/PAN-SPA, and PANSPA nanofibers were intertwined layer-by-layer in the interfacial transitional area, manifesting the outstanding bonding among the trilayers, which would decrease the water-transport resistance at the interface of the membranes. Fig. 2 depicts the SEM images of the three asprepared smooth and uniform nanofibrous membranes. The fiber diameters of the hydrophilic middle PU/PAN-SPA and outer PAN-SPA layers are approximately $152.3 \mathrm{~nm}$ and $122.5 \mathrm{~nm}$, respectively, but the fiber diameter of the hydrophobic PU inner layer is approximately $977.4 \mathrm{~nm}$. Abundant porous structures were formed by adjacent nanofibers in the nanofibrous membrane, and the average pore size of the membranes decreased in the following order: PU, PU/PAN-SPA, and PAN-SPA. The different pore sizes and surface wettability of the trilayered membranes endow them with the differential capillary effect, which is expected to improve the water-transport capacity.

\subsection{Influence of SPA content on properties of the middle nanofibrous} membrane

Figs. 3a and S1 show the change curves and images of the contact angles of the middle layer PU/PAN-SPA nanofibrous membrane with different SPA contents. The initial WCA of the PU/PAN-SPA membrane decreased from $130^{\circ}$ to below $90^{\circ}$ when the SPA content increased from 0 to $10 \mathrm{wt} \%$. At this time, the dynamic WCA of the PU/PAN-SPA nanofibrous membrane also rapidly decreased with an increase in SPA content. Fig. 3b shows that the time at which WCA of the nanofibrous membrane drops to zero decreases with an increase in SPA content.

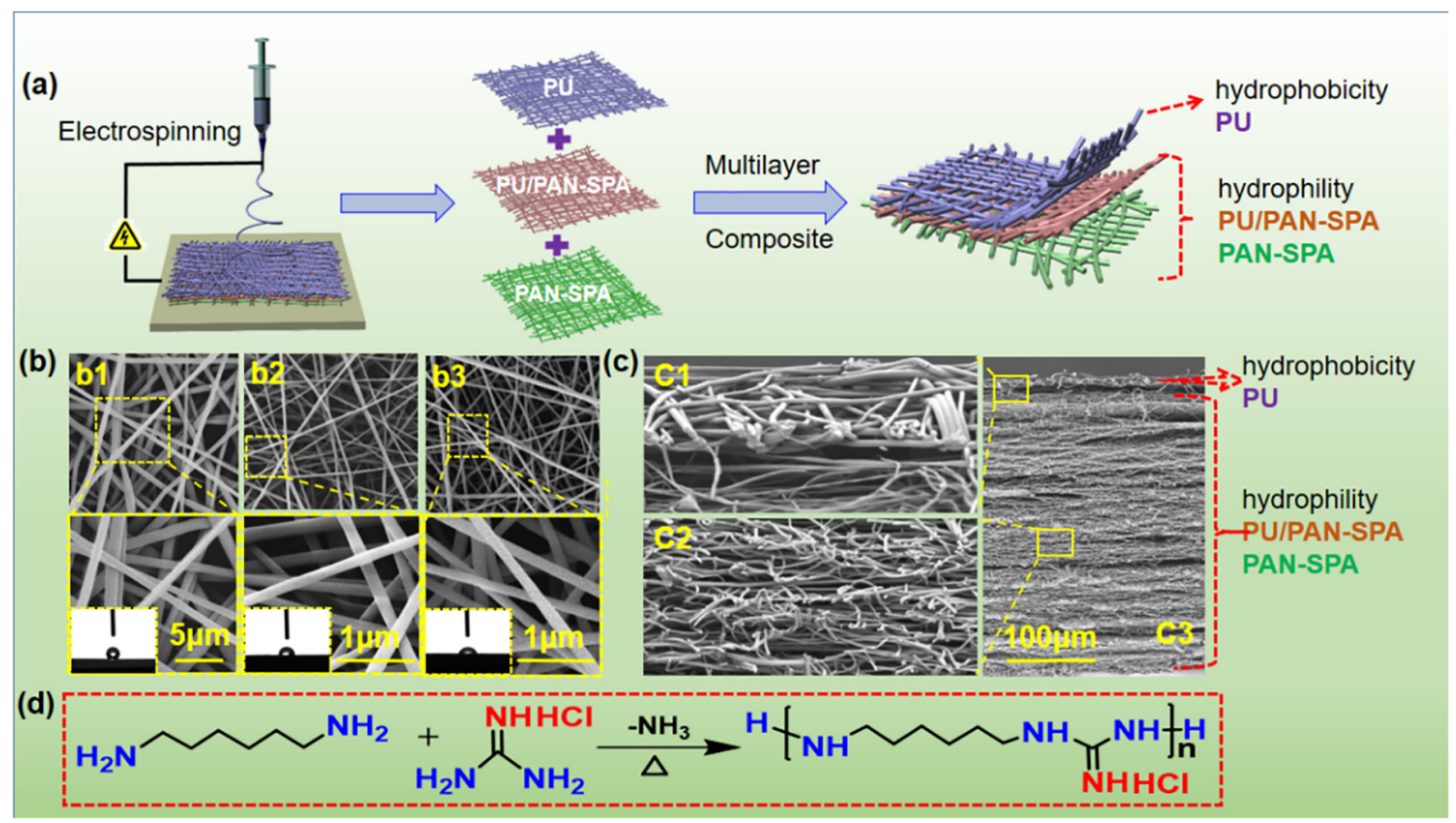

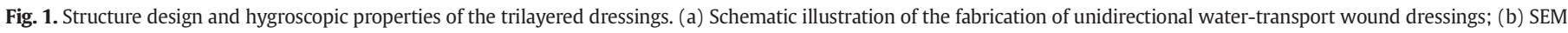

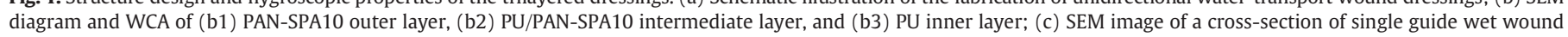
dressing; (d) Synthesis roadmap of PHCC macromolecular polymer. 


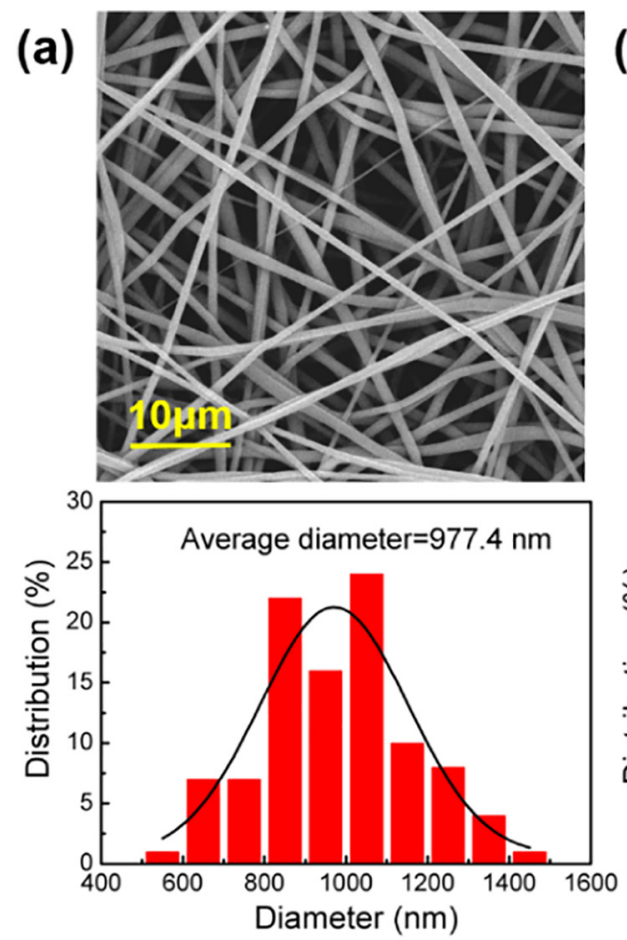

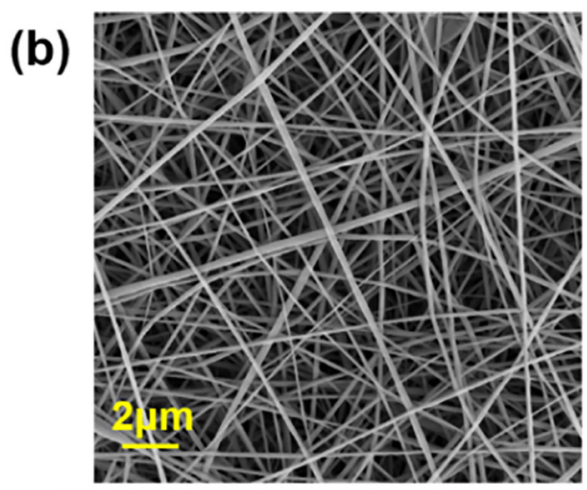

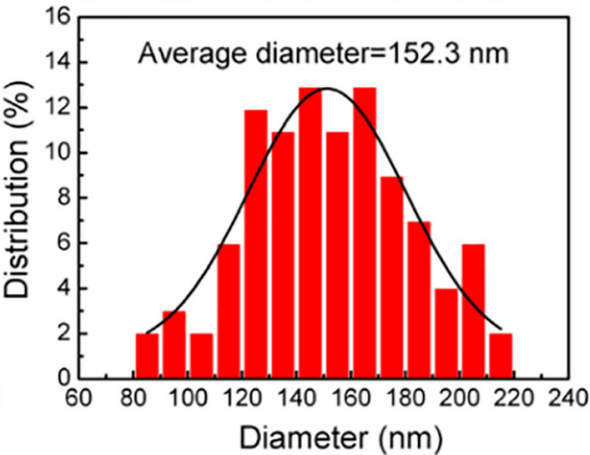

(c)
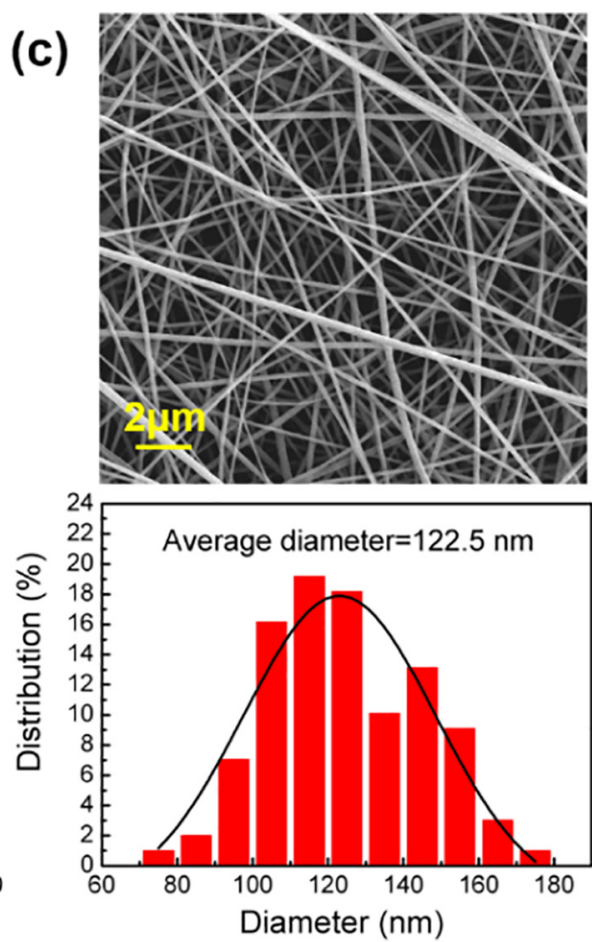

Fig. 2. SEM images and fiber diameter distribution of the (a) inner layer PU, (b) middle layer PU/PAN-SPA, and (c) outer layer PAN-SPA nanofibrous membranes.

When the SPA content was over $8 \mathrm{wt} \%$, the dynamic WCA of the nanofibrous membrane decreased rapidly to zero in $3 \mathrm{~s}$, because the presence of hydrophobic PU in the PU/PAN-SPA layer increases the initial WCA of nanofibrous membrane. Next, the droplet gradually spread, permeated into the membrane, and was quickly absorbed after contacting the super-hydrophilic SPA evenly dispersed in the fiber, resulting in a sharp decrease in the WCA of the fibrous membrane. In this process, the higher the SPA content, the faster the WCA of the nanofibrous membrane decreases. The results demonstrated that SPA could significantly improve the hydrophilicity of the nanofibrous membrane.

Fig. $3 c$ and d demonstrate that the water absorption and equilibrium water content of the PU/PAN-SPA nanofibrous membrane increased at first and then dropped with an increase in SPA content. When the SPA content increased to $10 \mathrm{wt} \%$, the water absorption and equilibrium water content of the nanofibrous membrane reached the maximum. This is because water-soluble SPA is uniformly dispersed in a spinning solution made of organic solvent. When the SPA content exceeds $10 \mathrm{wt} \%$, the dispersibility of the spinning solution worsens and can easily form precipitation, which ultimately affects the amount of SPA in the fiber membrane. Given the requirements of the SPA spinning solution, we chose $10 \mathrm{wt} \%$ SPA solution to conduct follow-up experiments. Under these circumstances, the water absorption and equilibrium water content of the nanofibrous membrane are up to $950 \%$ and $94 \%$, respectively, and the hydrophilicity of the membrane is optimum.

The wound dressing should have specific air and moisture permeability. Fig. 3e demonstrates the air and moisture permeability of the nanofibrous membranes. The air permeability of the nanofibrous membrane was maintained at $7 \mathrm{~mm} / \mathrm{s}$ with the SPA content from 0 to $12 \mathrm{wt} \%$. The WVTR gradually decreased from $2200 \mathrm{~g} / \mathrm{m}^{2} /$ day to $1930 \mathrm{~g} / \mathrm{m}^{2} /$ day with an increase in SPA content, indicating a linear relationship between the SPA content and WVTR. This is because air permeability is related to the structure of materials, and the WVTR is related to the surface properties of the fiber. The presence of SPA increases the hydrophilicity of the fiber membrane, but almost does not affect the membrane morphology. Thus, the air permeability of the fiber membrane is basically balanced. However, the escaped water vapor molecules are adsorbed on the surface of the fiber membrane with an increase in SPA content, leading to a decrease in the WVTR.

The middle layer of the trilayered dressing should have specific mechanical strength and ductility to support the upper and lower fiber membranes, so that the as-prepared wound dressing has suitable shape retention. A small amount of PU was added to PU/PAN-SPA layer to improve the mechanical properties of the middle layer. As shown in Figs. $3 \mathrm{f}$ and S2, the tensile strength and elongation at break of PU/PAN-SPA fiber membrane are in the range of 6-7.5 MPa and $40-50 \%$, respectively. The results demonstrate that added PU can markedly improve the mechanical properties of pure PAN fiber membrane. Therefore, the PU/PAN-SPA nanofiber membrane has appropriate toughness and mechanical strength, meeting the requirements of the ideal wound dressing. From the above, we chose $10 \mathrm{wt} \%$ SPA solution to fabricate the middle layer called PU/PAN-SPA10.

\subsection{Wettability of nanofibrous membrane and trilayered dressings}

The inner PU layer with different spinning time (PU- $x$ ), middle layer of PU/PAN-SPA10, and outer layer of the PAN-SPA10 nanofibrous membrane were combined to obtain the trilayered wound dressing. The wettability of three separate membranes and trilayered wound dressing with inner layer spinning times of $1 \mathrm{~h}, 2 \mathrm{~h}$, and $3 \mathrm{~h}$ are shown in Figs. 4 and S3. As shown in Fig. 4a, b, and d, the initial WCA of inner PU-1 membrane is $140^{\circ}$, while the initial WCAs of the middle and outer fiber membranes are less than $90^{\circ}$. There was no significant change in the WCA of the single PU-1 inner layer membrane in $10 \mathrm{~s}$. In contrast, the middle layer and outer fiber membrane contact angle rapidly dropped to zero at $1.16 \mathrm{~s}$ and $0.33 \mathrm{~s}$, respectively.

In addition, the comparison of the water absorption and equilibrium water content of the inner, middle, and outer membranes and the trilayered dressing with PU-1 membrane is illustrated in Fig. 4c. The equilibrium water content of the hydrophobic inner layer was 78\% and that of the other samples were maintained at $94 \%$, but the water absorption increased in the following order: from inner, middle, and outer membranes to trilayered dressings. These results demonstrated that there is a hydrophobic-hydrophilic gradient structure among the 

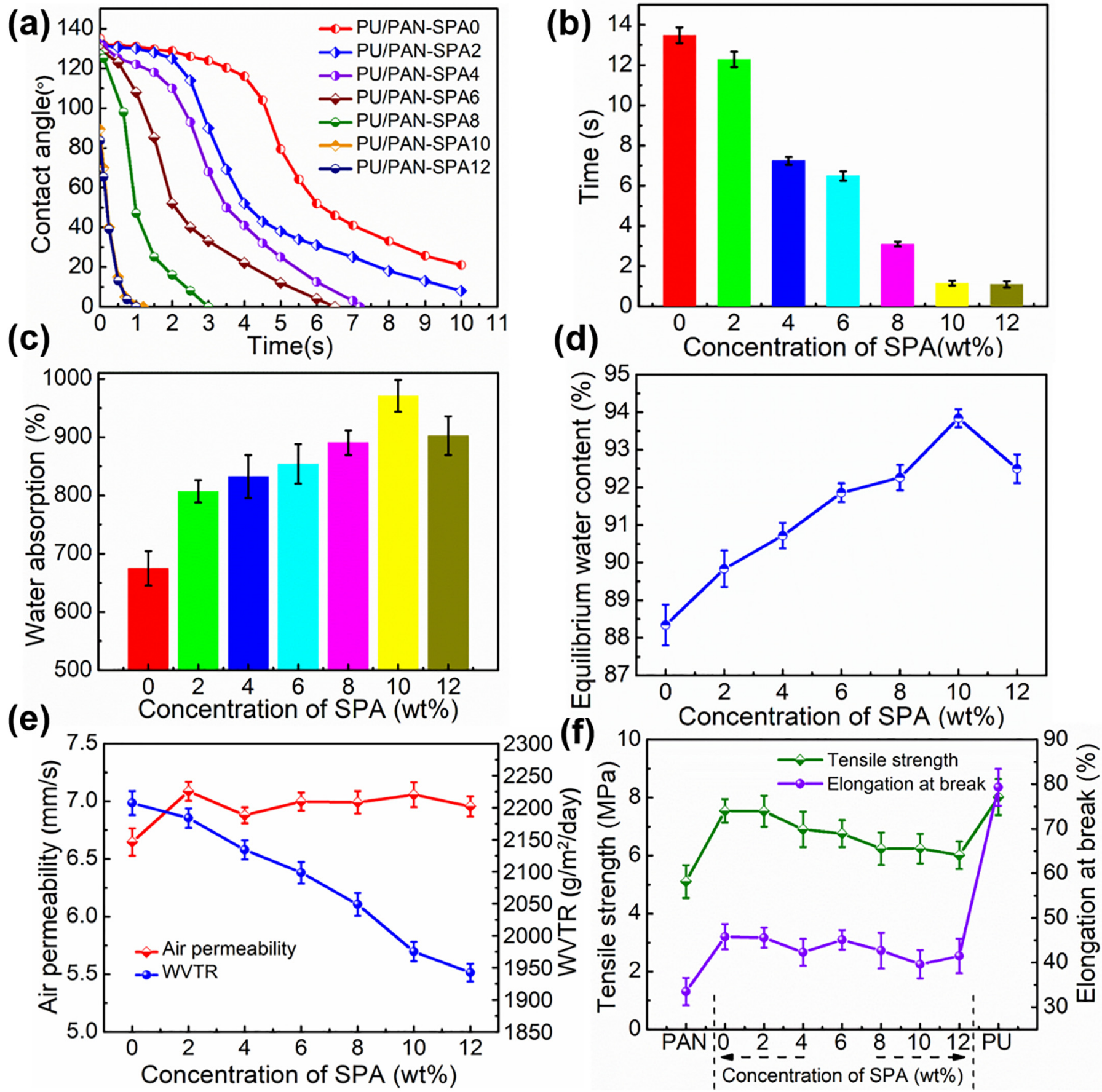

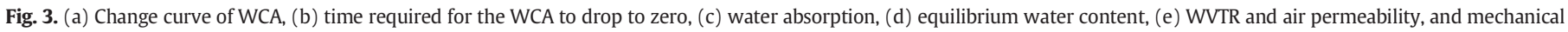
properties of PU/PAN-SPA nanofibrous membrane under different SPA contents.

inner, middle, and outer membranes of the trilayered dressing and that the hydrophilicity gradually increased from the inner to outer layer.

Because the inner layer of the trilayered dressing is directly in contact with the trauma, the biofluid needs to pass through the inner membrane before being absorbed by the middle and outer layer membranes with high hydrophilicity. Therefore, the thickness of the inner membrane has a direct impact on the biofluid absorption effect of the wound dressing. The inner membrane is too thin to block the exudate and too thick to drive wound exudate. The thickness of the inner layer membrane of the trilayered dressing could be controlled by the spinning time. We compared the WCA and the time taken by the WCA to drop to zero for the wound dressing with the spinning time of the PU inner membrane at $1 \mathrm{~h}, 2 \mathrm{~h}$, and $3 \mathrm{~h}$, respectively, as shown in Fig. 6. It can be observed that the thickness of the inner membrane increased, and the time required for the WCA of the dressing to drop to zero increases with an increase in spinning time.

PU-1 membrane was selected as the inner layer to fabricate the trilayered dressing to accelerate the drainage of the biofluid. Interestingly, the initial WCA can remain at $140^{\circ}$ for a single PU- 1 membrane, but the initial WCA becomes $130^{\circ}$ and the biofluid can be absorbed quickly within $2.2 \mathrm{~s}$ for the trilayered dressing. The biofluid flows from the inner to the outer layer to realize the unidirectional watertransport function. The principle will be explained in detail later. 
(a)

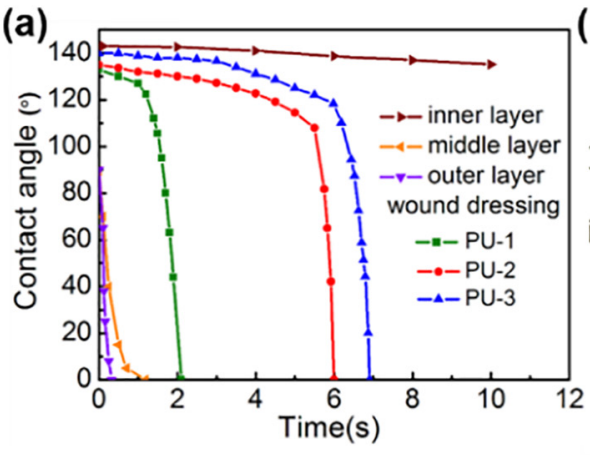

(b) 8

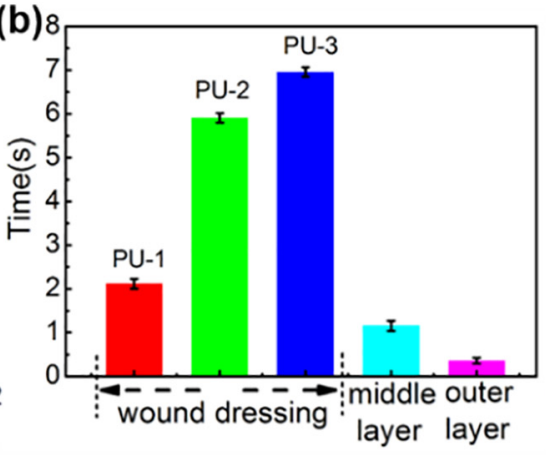

(c)

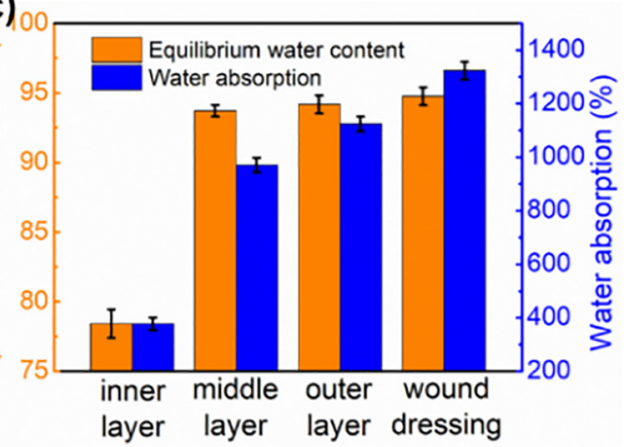

(d)

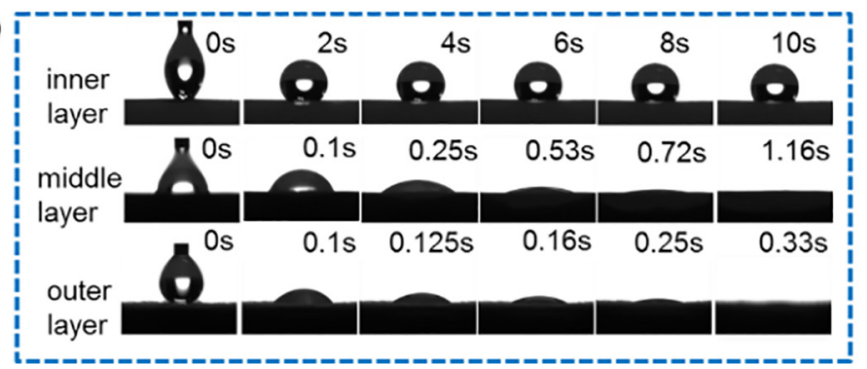

(e)

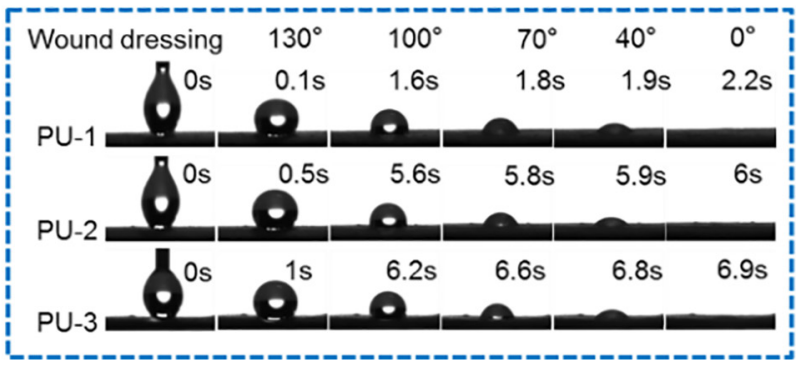

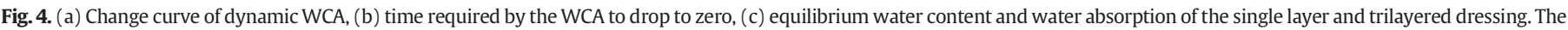
dynamic WCA picture of (d) inner PU-1, middle, and outer membrane, and (e) trilayered dressings with different PU spinning times.

3.5. Unidirectional water-transport properties of the nanofibrous membrane dressing

As demonstrated in Fig. $5 \mathrm{a}$ and b, $50 \mu \mathrm{L}$ blue ink droplets were added into the single-layer membrane and trilayered dressing with PU-1 membrane, and the diffusion diameter and time of ink droplet were recorded. The diffusion diameters of the ink droplet for the inner, middle, and outer layers as well as the trilayered dressing are 0.9, 4.2, 4.8, and $4.0 \mathrm{~cm}$, respectively. Because the single inner PU membrane is hydrophobic, the droplets hardly spread on the membrane within $200 \mathrm{~s}$. In contrast, the droplets can spread rapidly within the trilayered dressing. Fig. 5a and Movies S1 and S2 depict the diffusion area of ink droplets
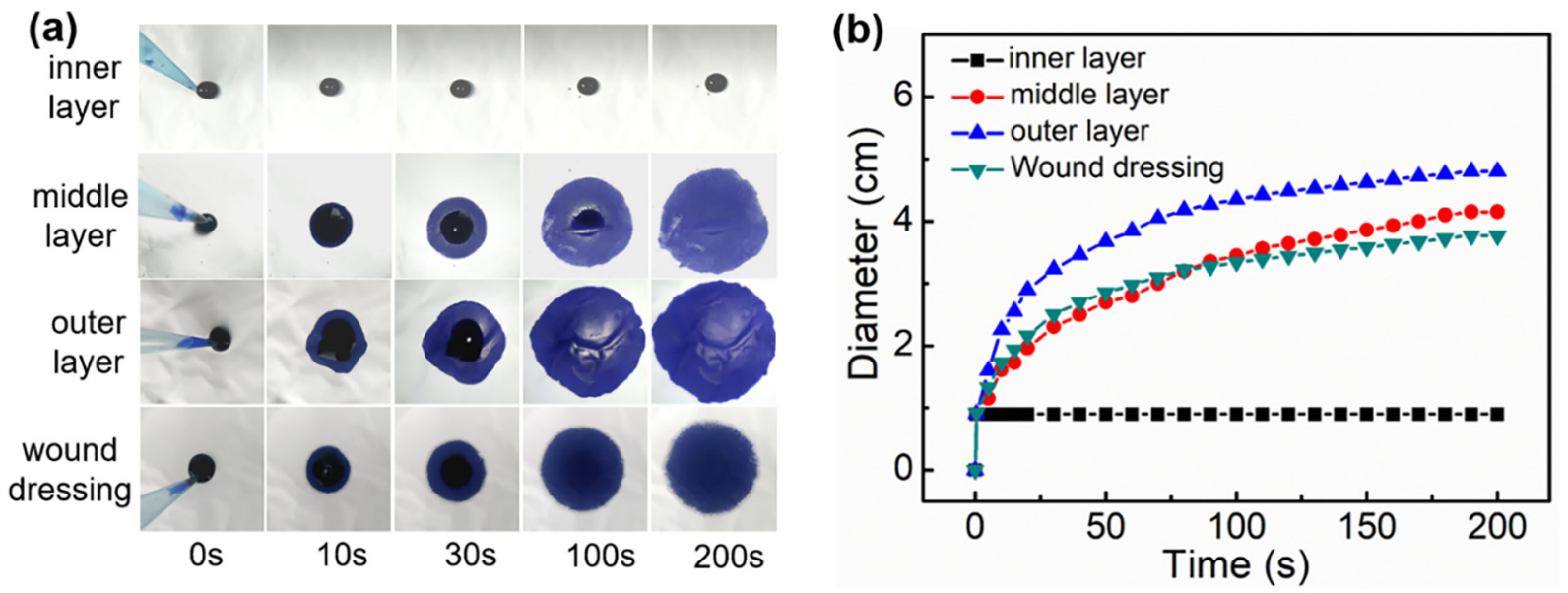

(c)

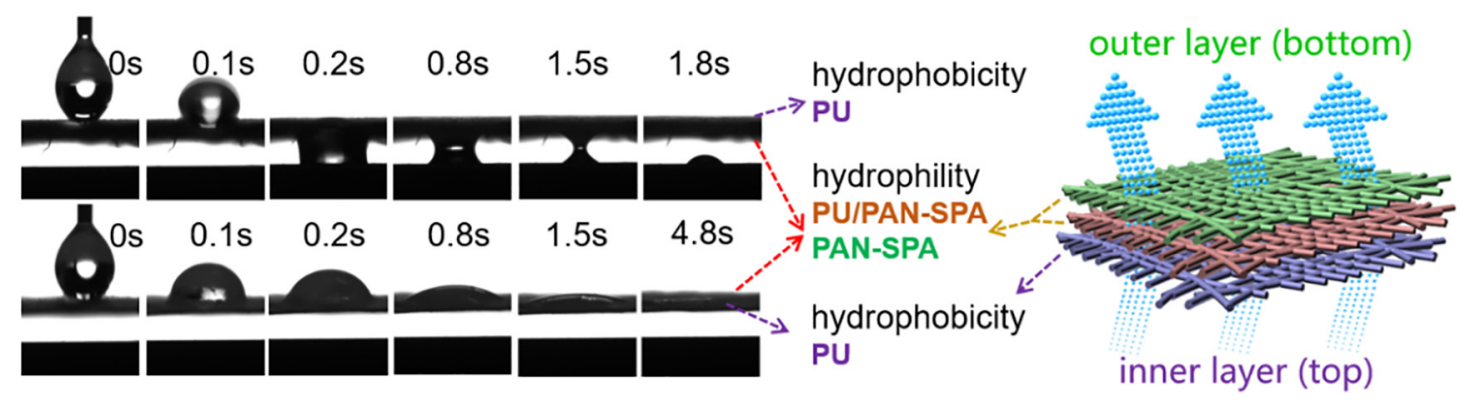

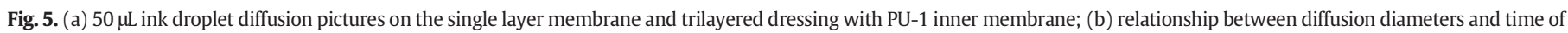
different membrane; (c) transmission process of $5 \mu \mathrm{L}$ water droplets on both sides of the dressing with PU- 1 inner membrane. 
Table 1

Key performance of the prepared trilayered dressing with PU-1 membrane.

\begin{tabular}{lllll}
\hline Sample & $\begin{array}{l}\text { Tensile strength } \\
(\mathrm{MPa})\end{array}$ & $\begin{array}{l}\text { Elongation at } \\
\text { break }(\%)\end{array}$ & $\begin{array}{l}\text { Air permeability } \\
(\mathrm{mm} / \mathrm{s})\end{array}$ & $\begin{array}{l}\text { WVTR } \\
\left(\mathrm{g} / \mathrm{m}^{2} / \text { day }\right)\end{array}$ \\
\hline Wound dressing & $6.7 \pm 0.6$ & $42 \pm 3$ & $6.8 \pm 0.5$ & $1360 \pm 30$ \\
\hline
\end{tabular}

on both sides of the trilayered dressing. The diffusion of the top surface (inner layer) is considerably smaller than the bottom surface (outer layer). The results demonstrate that the droplets in the trilayered dressing pass through the thickness direction from the inner to the outer layer and then quickly spread along the membrane surface. This also proves that the hydrophobic-hydrophilic gradient structure constructed among the inner, middle, and outer membranes of the trilayered dressing can effectively transport liquid to the outside of the wound.

As shown in Fig. 5c and Movies S3 and S4, $5 \mu \mathrm{L}$ water droplets were dropped on the top and bottom sides of the trilayered dressing, respectively. The transmission process of the droplets was recorded using a contact angle tester. The results demonstrate that water droplets dropped on the top side (inner layer) can be quickly absorbed and transferred to the other side via the dressing, while droplets dropped in the reverse direction are also quickly absorbed, but the presence of the PU membrane prevents the droplets from penetrating the other side, and Fig. S4 also supports the phenomenon. This proves that the constructed trilayered dressing with PU-1 membrane can unidirectionally drive the biofluid from the inner to the outer layer, preventing biofluid reverse osmosis and exhibiting excellent unidirectional watertransport performance.

To promote wound healing, the wound dressing should have specific mechanical strength and ductility to meet the requirements of clinical application. The literature has reported that the elastic modulus of human skin is between 4.6 and $20 \mathrm{MPa}$. Table 1 presents the key performance of the prepared trilayered wound dressing. The strength and elongation at break of the prepared trilayered dressing are $6.7 \pm$ $0.6 \mathrm{MPa}$ and $42 \pm 3 \%$, respectively, meeting the performance requirements of wound dressing. The activity of fibroblasts is related to the oxygen concentration; therefore, wound dressings are required to have a specific air and moisture permeability to provide a favorable microenvironment for wound repair. It is generally recognized that the optimal WVTR for moderate exudate wound dressings is in the range of $900-1450 \mathrm{~g} / \mathrm{m}^{2} /$ day. The WVTR of the prepared wound dressing is $1360 \pm 30 \mathrm{~g} / \mathrm{m}^{2} /$ day, which satisfies the requirements for wound healing.

\subsection{FTIR spectroscopy of PHGC and nanofibrous membrane}

To evaluate whether the synthesis of PHGC was successful, we added PHGC into the dressing and measured the TG curves of PHGC and wound dressing and the FTIR spectra of PHGC, PAN fiber membrane, and PAN-PHGC fiber membrane, as shown in Fig. 6. Fig. 6a illustrates that the thermal decomposition temperatures of the PHGC antibacterial agent and wound dressing prepared were $325^{\circ} \mathrm{C}$ and $275^{\circ} \mathrm{C}$, both with suitable thermal stability. In Fig. $6 \mathrm{~b}, 3300 \mathrm{~cm}^{-1}$ and $3180 \mathrm{~cm}^{-1}$ are stretching vibration peaks of the amino group $\mathrm{N}-\mathrm{H}$ in PHGC, $1660 \mathrm{~cm}^{-1}$ is a stretching vibration peak of guanidine group $\mathrm{C}=\mathrm{NH}^{+}$, and $1638 \mathrm{~cm}^{-1}$ is a bending vibration peak of the amine group $\mathrm{N}-\mathrm{H}$ in PHGC, which is a characteristic peak of the guanidine group, indicating the successful synthesis of PHGC $[38,39]$. The typical absorption peaks of pure PAN fibers at 2946, 2242, 1738, 1454, 1360, 1242, and $1075 \mathrm{~cm}^{-1}$ correspond to the contraction of $\mathrm{CH}_{2}, \mathrm{C} \equiv \mathrm{N}, \mathrm{C}=0$; bending deformation of $\mathrm{CH}_{2}$; vibration of $\mathrm{CH}_{2},-\mathrm{CH}$, and $\mathrm{C}-\mathrm{O}$, respectively [40]. These peaks are also present in the spectrum of the PAN-PHGC fiber; the peaks at 2933, 2860, and $2242 \mathrm{~cm}^{-1}$ appear weakened, indicating that PHGC was successfully added to the PAN nanofibrous membrane.

\subsection{Antibacterial properties of the nanofibrous dressing}

PHGC, as a polymer antibacterial agent, was added in the inner and outer layers of the wound dressing. The most common E. coli and S. aureus were selected as experimental strains. The nanofibrous membranes with different PHGC concentrations were placed in the same concentration of the bacterial solution. After culturing for $2 \mathrm{~h}, 0.1 \mathrm{~mL}$ of the bacterial solution was dropped on the agar medium and incubated at $37{ }^{\circ} \mathrm{C}$ for $12 \mathrm{~h}$. The antibacterial effects are shown in Fig. $6 \mathrm{c}$. The results demonstrate that the trilayered dressings with PHGC exhibit specific antibacterial action on both gram-negative and gram-positive bacteria, and the antibacterial performance of dressings strengthens with increasing PHGC concentration. When the PHGC concentration was $0.06 \mathrm{wt} \%$, almost no colonies were observed on the petri dishes of E. coli and S. aureus, which demonstrates that the optimal inhibitory concentration of PHGC in trilayered dressings is $0.06 \mathrm{wt} \%$.

In addition, the antibacterial effect of PHGC was tested by the bacteriostatic circle method, and the results are demonstrated in Fig. $6 \mathrm{~d}$. Fig. $6 \mathrm{~d} 1$ and $\mathrm{d} 2$ shows that $0.02 \mathrm{~g}$ of PHGC solid was directly placed into a petri dish to test its antibacterial activity against $E$. coli and $S$. aureus. The results demonstrate a distinct inhibition zone with a diameter of $3 \mathrm{~cm}$, which proves that PHGC has suitable antibacterial properties. However, when testing the antibacterial properties of the inner and outer membranes with PHGC concentrations of 0 and 0.06 wt\%, there was no distinct inhibition zone on the petri dish. Compared with the membranes without PHGC, there was no bacterial growth on the back of the membrane with 0.06 wt\% PHGC, indicating that PHGC and the prepared membrane with PHGC exhibit appropriate antibacterial properties. The membranes with PHGC do not exhibit a distinct inhibition zone, which can be explained as follows: PHGC, as a macromolecule polymer, can be retained in the fibrous membrane for a long time, and it is difficult to dissolve out from the membrane; hence, PHGC is a nondissolution antibacterial agent. The fabricated dressings with PHGC possess long-term antimicrobial properties, and PHGC is safe for humans because it cannot enter the skin wound.

\subsection{In vitro cytotoxicity of nanofibrous membrane dressings}

The cytotoxicity of single or trilayered membranes as wound dressings was evaluated using the methodology of MTT conversion into formazan crystals by means of viable cells. Fig. 7a and b show the cell viability of GES- 1 for all the samples. The results demonstrate that the viabilities of GES- 1 are above $95 \%$ after incubation $24 \mathrm{~h}$ and $48 \mathrm{~h}$ in control and extracts from membranes. These results indicate that all the membranes did not cause any cytotoxic effect to the GES-1 cells in the periods of time assayed, and the trilayered nanofibrous dressings possess good biocompatibility and are promising candidate dressings in wound repair.

An ideal wound dressing should have low cell adhesion to wound sites. The in vitro bioadhesion behavior of wound dressings was evaluated to study the cellular interactions with nanofiber-based dressings. As shown in Fig. 7c and d, the cell morphology and adhesion on the surface of PU inner layer and PAN-SPA outer layer membrane were characterized using SEM. After 2 days of incubation, it was clearly observed that cells on the sample surface showed spherical shape, and more cells attached to the surface of PAN-SPA than PU membrane, illustrating that inner layer PU membrane had lower adhesion than the outer layer membrane of trilayered dressings. During dressing replacement, thanks to the low cell adhesion performance of the inner PU nanofibrous membrane, the wound pain can be effectively avoided, and the new granulation tissue will not be destroyed.

\subsection{Unidirectional water-transport mechanisms}

Further investigation of the unidirectional water-transport mechanisms of trilayered nanofibrous membranes with hydrophobic-hydrophilic gradient structures would be helpful for 
(a)

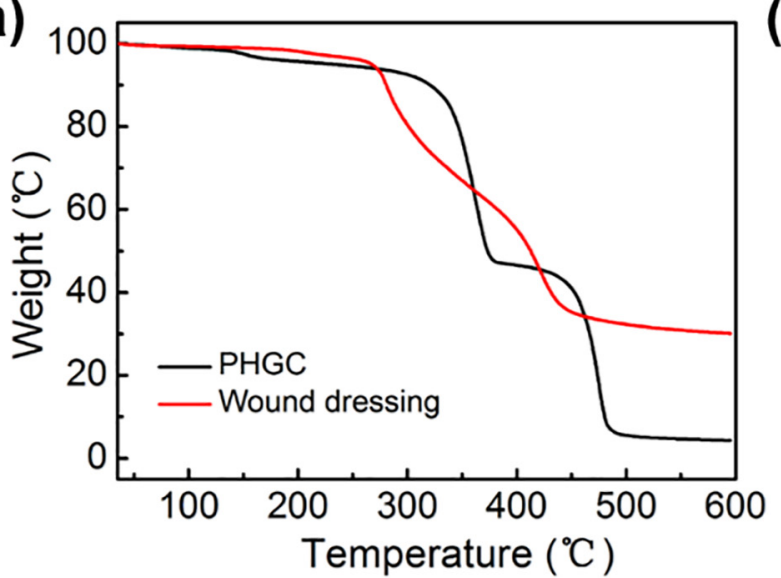

(b)

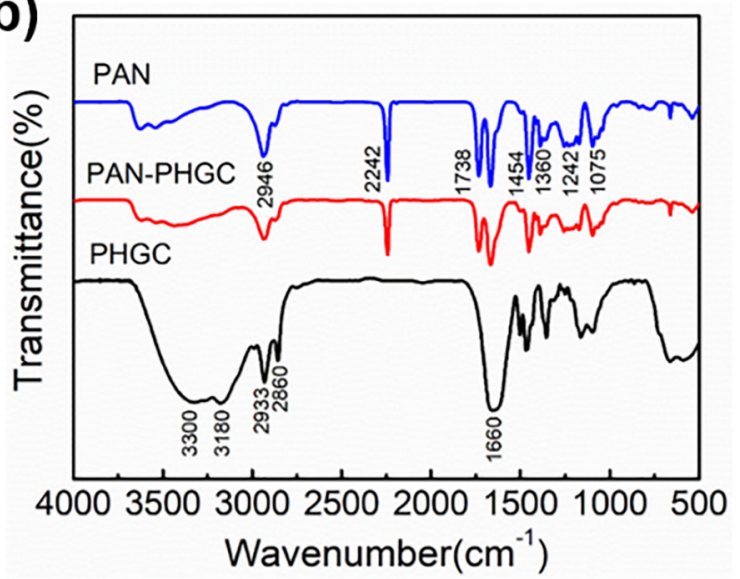

(c)

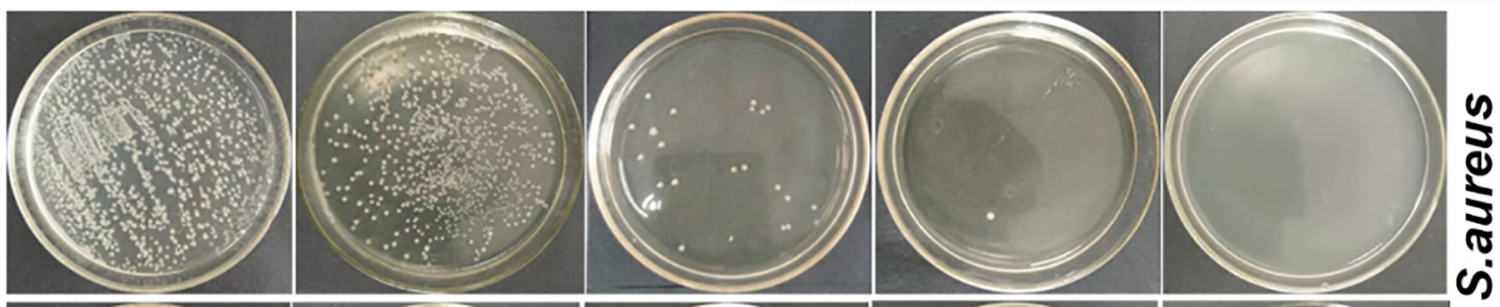

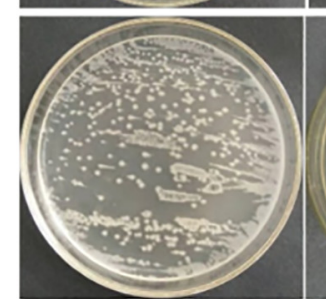

Owt $\%$

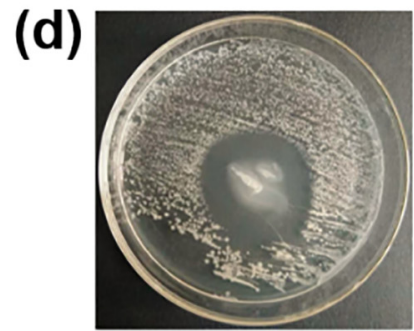

d1

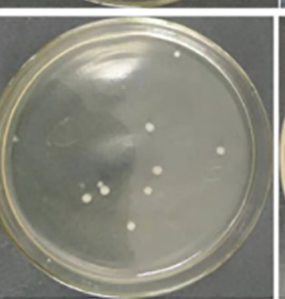

$0.02 w t \%$

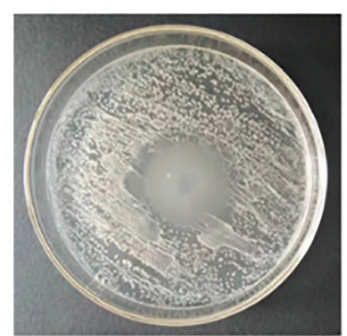

d2

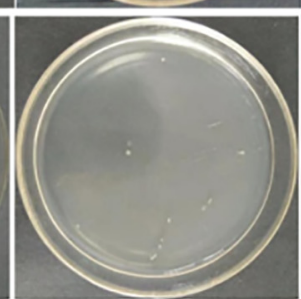

$0.04 w t \%$

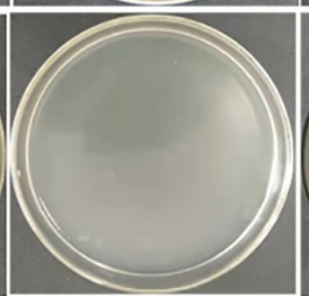

$0.06 w t \%$

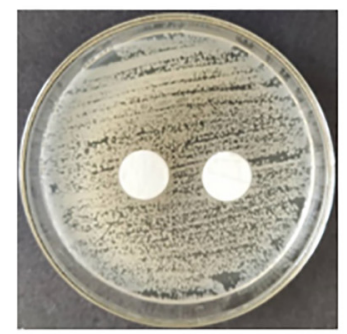

d3

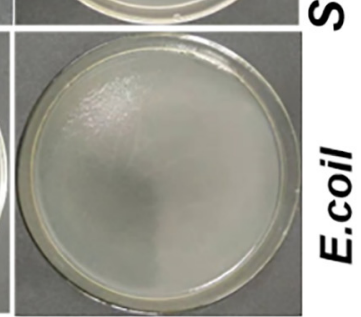

$0.08 w t \%$

d4

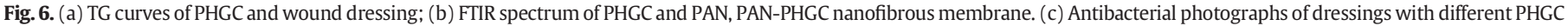

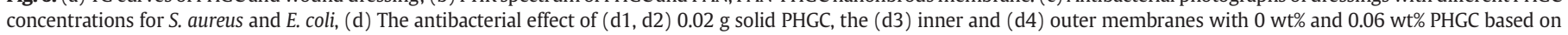
inhibition zone methods. (d1, d3) S. aureus, (d2, d4) E. coli.

the rational design of wound dressings. The pore sizes and WCA of the trilayered membranes decreased from the inner layer to the outer layer, resulting in unidirectional water-transport behavior owing to an increase in capillary force. Drawing upon the above discussion, the unidirectional water-transport behavior can be elucidated based on the following theoretical model (Fig. 8).

For biofluid-transport in the single-layer membrane, the droplet can spontaneously penetrate the membranes in the presence of capillary force. Obviously, the capillary force originates from the Laplace pressure $P_{L}$, which can be calculated according to the Young-Laplace Equation:

$P_{L}=\frac{4 \gamma \times \cos \theta}{D}$

where $\theta, \gamma$, and $D$ denote the static contact angle on the nanofibrous membrane, surface tension of water in the air, and diameter of the capillaries, respectively. From Eq. 4, the capillary force was inversely proportional to the pore size and WCA, indicating that a smaller pore size and higher WCA of nanofiber membranes can result in higher $P_{L}$. The pore size and WCA of the three-layer membrane in wound dressings decrease according to the following order: inner layer, middle layer, and outer layer. Consequently, the outer layer holds the optimum capillary force, and the inner layer exhibited the lowest capillary force. The different porous structures can generate synergetic capillary pressure difference $(\Delta P)$, as follows:

$\Delta P_{1}=P_{L 2}-P_{L 1}=\frac{4 \gamma \times \cos \theta_{2}}{D_{2}}-\frac{4 \gamma \times \cos \theta_{1}}{D_{1}}$

$\Delta P_{2}=P_{L 3}-P_{L 2}=\frac{4 \gamma \times \cos \theta_{3}}{D_{3}}-\frac{4 \gamma \times \cos \theta_{2}}{D_{2}}$

where $D_{1}, D_{2}$, and $D_{3}$ are the diameters of the inner, middle, and outer layer pores; and $\theta_{1}, \theta_{2}$, and $\theta_{3}$ are the corresponding surface WCA of the inner, middle, and outer layer membranes, respectively. Considering that $\cos \theta_{3}>\cos \theta_{2}>\cos \theta_{1}$ and $D_{1}>D_{2}>D_{3}$, the capillary pressure of 

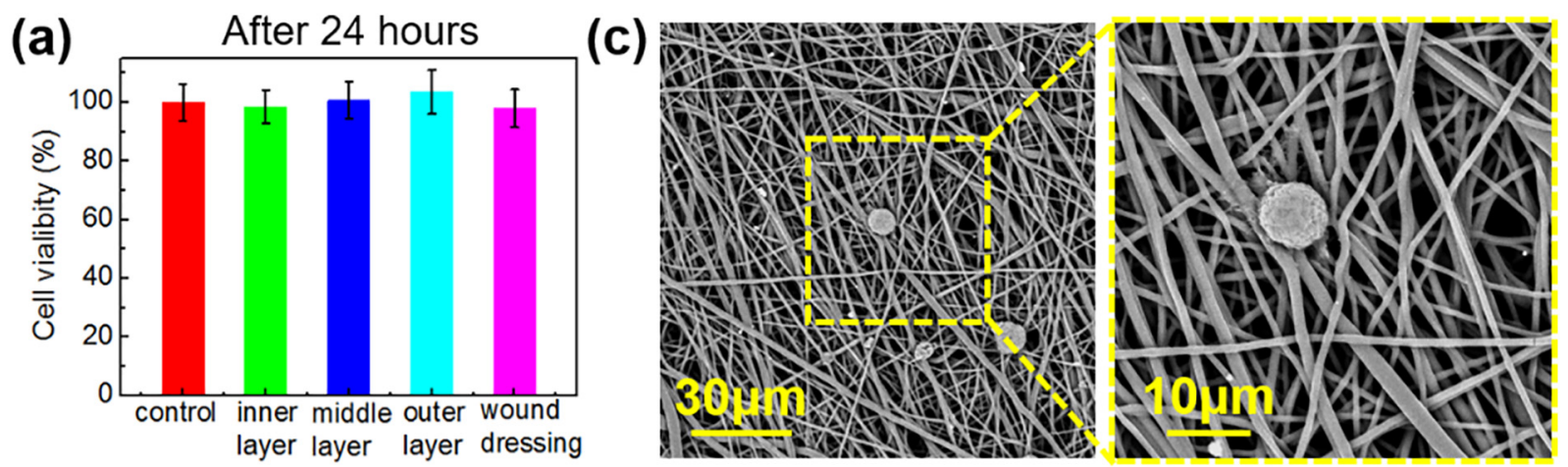

(b)

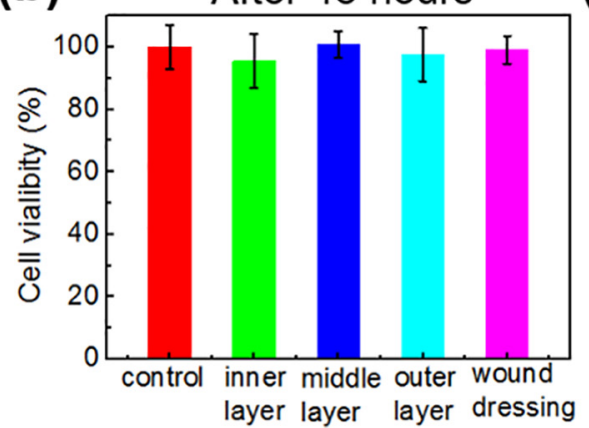

(d)

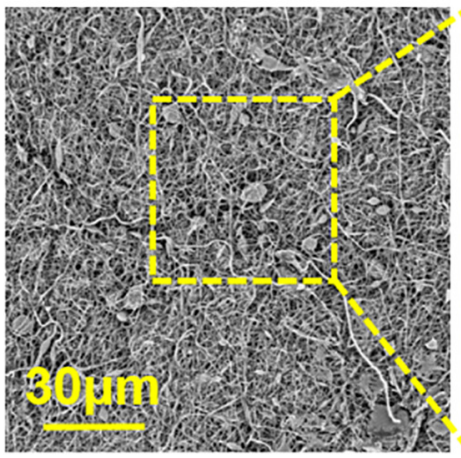

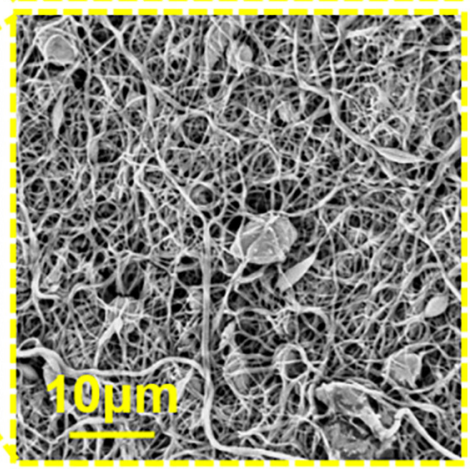

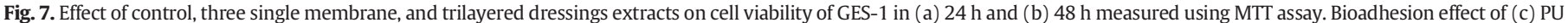
inner layer and (d) PAN-SPA outer layer surface of trilayered dressings for culturing $48 \mathrm{~h}$.

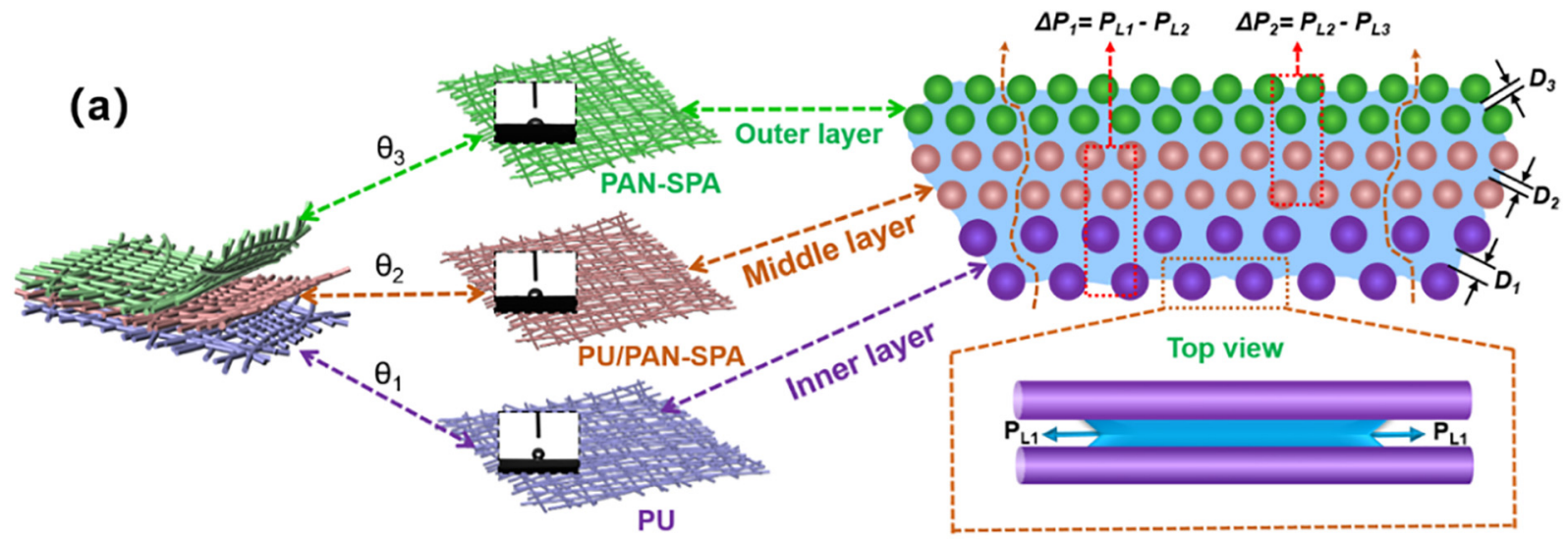

(b)

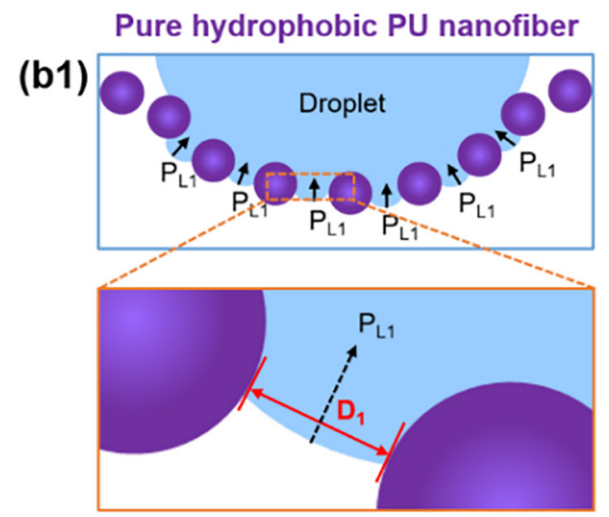

Hydrophobic PU
Self-pumping nanofibrous membrane

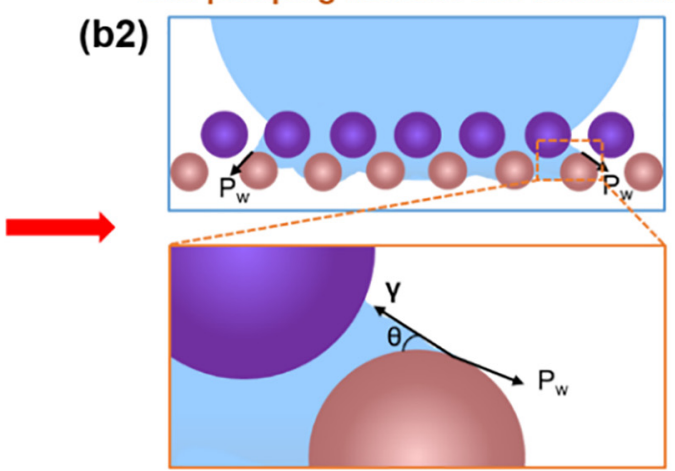

Hydrophilic PU/PAN-SPA

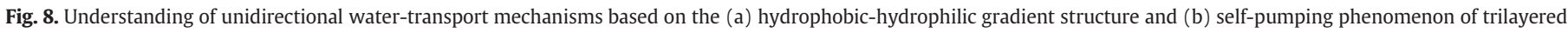

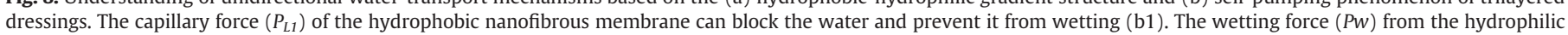
nanofibrous membrane of the self-pumping dressing could drain the droplets from the contacting points (b2). 
the trilayered membrane in wound dressings decreases according to the following order: $P_{L 3}>P_{L 2}>P_{L 1}$. Thus, $\Delta P$ plays a significant role in the driving force that extracts water directly from the inner layer to the outer layer. This mechanism is in accordance with Murray's law.

In addition, the water droplet presented a perfect spherical shape (WCA $>130^{\circ}$ ) after dropping it on the pure hydrophobic PU nanofibrous membrane (Fig. 8b1). However, when the droplet contacts the hydrophobic-hydrophilic bilayered nanofibrous membrane, the hydrophilic layer can be wetted rapidly through its hydrophobic layer (Fig. 8b2). This phenomenon demonstrates that the hydrophobichydrophilic bilayered nanofibrous membrane can construct a selfpumping dressing that can drive the water-transport unidirectionally from the hydrophobic side to the hydrophilic side.

In the bilayered or trilayered self-pumping dressing, the hydrophilic and hydrophobic nanofibers formed multiple hydrophobic-hydrophilic contact points. When the water droplet contacted the hydrophobic nanofibers at multiple contact points, the wetting force from the hydrophilic nanofibers would pump water wetting the hydrophilic nanofibers. In this process, the wetting force $P_{W}$ can be calculated based on Eq. 7:

$P_{W}=\gamma p \cos \theta$

where $p$ represents the perimeter of the contact line.

For the pure hydrophobic PU nanofibrous membrane, $P_{L 1}$ from hydrophobic nanofibers can hold water and keep it from penetrating the hydrophobic nanofibrous membrane. For the designed self-pumping nanofibrous membranes, water droplets can contact the hydrophilic nanofibers through multiple contact points, and $P_{w}$ from the hydrophilic nanofibers can significantly reduce the hydrostatic pressure compared to the corresponding pure hydrophobic nanofibrous membrane. Above all, we propose that the self-pumping phenomenon and hydrophobic-hydrophilic gradient structure contribute to the unidirectional water-transport wound dressings.

\section{Conclusions}

In summary, an antibacterial low-adherent trilayered nanofibrous wound dressing with unidirectional water-transport function was fabricated using electrospinning technology by introducing macromolecular antibacterial PHGC and adjusting the surface properties and thickness of each nanofibrous membrane. The resulting trilayered dressings exhibited excellent unidirectional water-transport function owing to the presence of a hydrophobic-hydrophilic gradient structure. The trilayered dressings also exhibited superior water absorption; appropriate toughness and ductility; low cell adhesion; and suitable biocompatibility as well as air and moist permeability for wound healing. In addition, the trilayered wound dressing with 0.06 wt\% PHGC macromolecular antibacterial agent exhibited excellent long-term antibacterial properties compared to those with low or no PHGC. The unidirectional water-transport mechanisms of dressings can be attributed to the hydrophobic-hydrophilic gradient structure and self-pumping effect, which can rapidly and unidirectionally drive the biofluids outflow and prevent the wound exudates from reverse osmosis, providing a new strategy for the preparation of wound care products.

\section{CRediT authorship contribution statement}

Linya Qi: Data curation, Writing - original draft, Formal analysis. Kangkang Ou: Methodology, Writing - review\&editing, Supervision. Yijun Hou: Investigation, Validation. Pingping Yuan: Validation, Formal analysis. Wen Yu: Investigation, Formal analysis. Xiang Li: Writing - review\&editing. Baoxiu Wang: Writing - review \&editing. Jianxin He: Resources, Methodology, Supervision. Shizhong Cui: Methodology, Supervision. Xiaogang Chen: Conceptualization.

\section{Declaration of Competing Interest}

The authors declare that they have no known competing financial interests or personal relationships that could have appeared to influence the work reported in this paper.

\section{Acknowledgements}

This work was supported by a grant from the National Key Research and Development Program of China (2020YFC0844800), National Natural Science Foundation of China (No. 52003048; 11905307; 51803244), Henan Province Science and Technology Research Project (No. 202102210060; 212102210041), Key Scientific Research Project in Universities of Henan Province (No. 20A540003; 20B540003), and the Project for Fundamental Research Funds of Zhongyuan University of Technology (K2019QN003). The Science and Technology Guidance Project of China Textile Industry Federation (No. 2019028; 2019007) is also gratefully acknowledged. We would like to thank Editage (www. editage.cn) for English language editing.

\section{Appendix .A}

Supplementary data to this article can be found online at https://doi. org/10.1016/j.matdes.2021.109461.

\section{References}

[1] L.X. Shi, X. Liu, W.S. Wang, L. Jiang, S.T. Wang, A self-pumping dressing for draining excessive biofluid around wounds, Adv. Mater. 31 (5) (2019) 1804187.

[2] T.T. Cui, J.F. Yu, Q. Li, C.F. Wang, G.F. Wang, Large-scale fabrication of robust artificial skins from a biodegradable sealant-loaded nanofiber scaffold to skin tissue via microfluidic blow-spinning, Adv. Mater. 32 (32) (2020) 2000982.

[3] K.K. Ou, X.J. Wu, B.X. Wang, C. Meng, X. Dong, H. Jinxin, Controlled in situ graft polymerization of DMAEMA onto cotton surface via SI-ARGET atrp for low-adherent wound dressings, Cellulose 24 (11) (2017) 5211-5224.

[4] K.S. Liu, X. Yao, L. Jiang, Recent developments in bio-inspired special wettability, Chem. Soc. Rev. 39 (8) (2010) 3240-3255.

[5] Z.J. Wang, Y. Wan, G.J. Li, Rapid and efficient separation of oil from oil-in-water emulsions using a Janus cotton fabric, Angew. Chem. Int. Ed. 55 (4) (2016) 1291-1294.

[6] K. Yin, S. Yang, X.R. Dong, D.K. Cui, J.A. Duan, J. He, Ultrafast achievement of a superhydrophilic/hydrophobic Janus foam by femtosecond laser ablation for directional water transport and efficient fog harvesting, ACS Appl. Mater. Interfaces 10 (37, 2018) 31433-31440.

[7] Y.M. Zheng, H. Bai, Z.B. Huang, X.L. Tian, F.Q. Nie, Y. Zhao, J. Zhai, L. Jiang, Directional water collection on wetted spider silk, Nature 463 (7281) (2010) 640-643.

[8] X.F. Wang, Z. Huang, D.Y. Miao, J. Zhao, J.Y. Yu, B. Ding, Biomimetic fibrous Murray membranes with ultrafast water transport and evaporation for smart moisturewicking fabrics, ACS Nano 13 (2) (2019) 1060-1070.

[9] J.B. You, Y. Yoo, M.S. Oh, S.G. Im, Simple and reliable method to incorporate the Janus property onto arbitrary porous substrates[J], ACS Appl. Mater. Interfaces 6 (6) (2014) 4005-4010.

[10] L.L. Hou, N. Wang, X.K. Man, Z.M. Cui, J. Wu, J.C. Liu, S. Li, Y. Gao, D.M. Li, L. Jiang, Y. Zhao, Interpenetrating Janus membrane for high rectification ratio liquid unidirectional penetration, ACS Nano 13 (2019) 4124-4132.

[11] R.F. Zhu, M.M. Liu, Y.Y. Hou, L.P. Zhang, M. Li, D. Wang, D. Wang, S.H. Fu, Biomimetic fabrication of Janus fabric with asymmetric wettability for water purification and hydrophobic/hydrophilic patterned surfaces for fog harvesting, ACS Appl. Mater. Interfaces 12 (44) (2020) 501130-501205.

[12] S.C.M. Fernandes, P. Sadocco, A.V. Ana, P. Teodoro, E. Arantxa, A.J.D. Silvestre, I. Mondragon, C.S.R. Freire, Bioinspired antimicrobial and biocompatible bacterial cellulose membranes obtained by surface functionalization with aminoalkyl groups, ACS Appl. Mater. Interfaces 5 (8) (2013) 3290-3297.

[13] J.W. Yao, Q.T. Fang, G.L. Zhang, C.C. Yang, K.M. Niu, Effect of hydrophilic-hydrophobic ratio in self-emulsifying amphiphilic epoxy sizing agent on interfacial properties of carbon fibre/epoxy composites, Prog. Org. Coat. 143 (2020), 105621,

[14] L.S. Zou, P. Gusnawan, G.Y. Zhang, J.J. Yu, Study of the effective thickness of the water-intrudable hydrophilic layer in dual-layer hydrophilic-hydrophobic hollow fiber membranes for direct contact membrane distillation, J. Membr. Sci. 615 (2020) 118552.

[15] E. Schoolaert, L. Cossu, J. Becelaere, J.F.R. Van Guyse, K. De Clerck, Nanofibers with a tunable wettability by electrospinning and physical crosslinking of poly(2-n-propyl2-oxazoline), Mater. Des. 192 (2020) 108747.

[16] C. Amit, A. Jaideep, G. Aritri, C. Sougata Roy, T. Sabu, D. Pallab, S. Prosenjit, Electrospun chitosan/polycaprolactone-hyaluronic acid bilayered scaffold for potential wound healing applications, Int. J. Biol. Macromol. 116 (2018) 774-785. 
[17] K. Ghosal, C. Agatemor, Z. pitálsky, S. Thomas, E. Kny, electrospinning tissue engineering and wound dressing scaffolds from polymer-titanium dioxide nanocomposites, Chem. Eng. J. 358 (2019) 1262-1278.

[18] G.M. Wu, X. Ma, L. Fan, Y.Y. Gao, Y.N. Wang, Accelerating dermal wound healing and mitigating excessive scar formation using LBL modified nanofibrous mats, Mater. Des. 185 (2020), 108265, .

[19] Y.X. Wang, H. Tao, M.N. Chen, C.T. Chang, Core-sheath fiber-polyurethane guided solid particles grafted on polyacrylonitrile fiber surface and its application in adsorbing acetone, Microporous Mesoporous Mater. 308 (2020) 110252.

[20] W. Yang, R. Li, C.P. Fang, W.T. Hao, Surface modification of polyamide nanofiber membranes by polyurethane to simultaneously improve their mechanical strength and hydrophobicity for breathable and waterproof applications, Prog. Org. Coat. 131 (2019) 67-72.

[21] C.H. Yao, C.Y. Lee, C.H. Huang, Y.S. Chen, K.Y. Chen, Novel bilayer wound dressing based on electrospun gelatin/keratin nanofibrous mats for skin wound repair, Mater. Sci. Eng. C 79 (2017) 533-540.

[22] D. Wang, Y.Y. Yue, Q.X. Wang, W.L. Cheng, G.P. Han, Preparation of cellulose acetatepolyacrylonitrile composite nanofibers by multi-fluid mixing electrospinning method: morphology, wettability, and mechanical properties, Appl. Surf. Sci. 510 (2020) 145462.

[23] M. Khayet, C. García-Payo, T. Matsuura, Superhydrophobic nanofibers electrospun by surface segregating fluorinated amphiphilic additive for membrane distillation, J. Membr. Sci. 588 (2019) 117215.

[24] C.M. Liu, H.C. Shi, H.W. Yang, S.J. Yan, S.F. Luan, Y.C. Li, M.Y. Teng, A.F. Khan, J.H. Yin, Fabrication of antibacterial electrospun nanofibers with vancomycin-carbon nanotube via ultrasonication assistance, Mater. Des. 120 (2017) 128-134.

[25] L.I.F. Moura, A.M.A. Dias, E. Carvalho, H.C. de Sousa, C. Hermínio, Recent advances on the development of wound dressings for diabetic foot ulcer treatment-a review, Acta Biomater. 9 (7) (2013) 7093-7114.

[26] C.A.W. Choong, K.Y. Cheong, T.B. Keem, T.K. Chai, F.C. Liam, C.S. Jack, Skin tissue engineering advances in severe burns: review and therapeutic applications, Burns Trauma 4 (1) (2016) 3.

[27] J.K. Yang, K. Wang, D.-G. Yu, Y.Y. Yang, G.R. Williams, Electrospun janus nanofibers loaded with a drug and inorganic nanoparticles as an effective antibacterial wound dressing, Mater. Sci. Eng. C 111 (4) (2020) 110805.

[28] ZJ. Jia, P. Xiu, M. Li, X.C. Xu, Y.Y. Shi, Y. Cheng S.C. Wei, Y.F. Zheng T.F. Xi, H. Cai, Z.J Liu, Bioinspired anchoring agnps onto micro-nanoporous $\mathrm{TiO}_{2}$ orthopedic coatings: trap-killing of bacteria, surface-regulated osteoblast functions and host responses, Biomaterials 75 (2016) 203-222.
[29] M.R. Michael, L. Anupama, A.T.K. Ping, C.M. Jia, C.A.E. Hauser, In situ synthesis of size-controlled, stable silver nanoparticles within ultrashort peptide hydrogels and their anti-bacterial properties, Biomaterials 35 (26) (2014) 7535-7542.

[30] D.H. Liang, Z. Lu, H. Yang, J.T. Gao, R. Chen, Novel asymmetric wettable AgNPs/chitosan wound dressing: in vitro and in vivo evaluation, ACS Appl. Mater. Interfaces 8 (6) (2016) 3958-3968.

[31] S. Homaeigohar, A.R. Boccaccini, Antibacterial biohybrid nanofibers for wound dressings, Acta Biomater. 107 (2020) 25-49.

[32] H.N. Xing, M. Lu, T.Z. Yang, H. Liu, Y.P. Sun, X.Y. Zhao, H. Xu, L. Yang, P.T. Ding, Structure-function relationships of nonviral gene vectors: lessons from antimicrobial polymers, Acta Biomater. 86 (2019) 15-40.

[33] M.O. Gabriela, C. Luciana, S. Milena, R. Jacqueline, A.R. Viviana, V.I. Sabrina, Polyhexamethylene guanidine as a fungicide, disinfectant and wound protector in lemons challenged with penicillium digitatum, Food Microbiol. 76 (2018) 128-134.

[34] L.L. Zhu, L. Wang, X.Q. Zhang, T. Li, Interfacial engineering of graphenic carbon electrodes by antimicrobial polyhexamethylene guanidine hydrochloride for ultrasensitive bacterial detection, Carbon 159 (2020) 185-194

[35] M. Sahraro, H. Yeganeh, M. Sorayya, Guanidine hydrochloride embedded polyurethanes as antimicrobial and absorptive wound dressing membranes with promising cytocompatibility, Mater. Sci. Eng. C 59 (2016) 1025-1037.

[36] H. Han, J. Zhu, D.Q. Wu, F.X. Li, X.L. Wang, J.Y. Yu, X.H. Qin, Inherent guanidine nanogels with durable antibacterial and bacterially antiadhesive properties, Adv. Funct. Mater. 29 (12) (2019) 1806594

[37] M. Ochoa, R. Rahimi, J.E. Zhou, H.J. Jiang, C.K. Yoon, D. Maddipatla, B.B. Narakathu, V. Jain, M.M. Oscai, T.J. Morken, R.H. Oliveira, G.L. Campana, O.W. Cummings, M.A. Zieger, R. Sood, M.Z. Atashbar, B. Ziaie, Integrated sensing and delivery of oxygen for next-generation smart wound dressings, Microsyst. Nanoeng. 6 (1) (2020) 46.

[38] J. Chen, D.F. Wei, W.L. Gong, A.N. Zheng, Y. Guan, Hydrogen-bond assembly of poly (vinyl alcohol) and polyhexamethylene guanidine for nonleaching and transparent antimicrobial films, ACS Appl. Mater. Interfaces 10 (43) (2018) 37535-37543.

[39] P. Li, S.Y. Sun, A. Dong, Y.P. Hao, S.Q. Shi, Z.J. Sun, G. Gao, Y.X. Chen, Developing of a novel antibacterial agent by functionalization of graphene oxide with guanidine polymer with enhanced antibacterial activity, Appl. Surf. Sci. 355 (15) (2015) 446-462.

[40] H.J. Chen, J.X. Yang, Q. Shuai, J. Li, Q. Ouyang, S. Zhang, In-situ doping $\mathrm{B}_{4} \mathrm{C}$ nanoparticles in PAN precursors for preparing high modulus PAN-based carbon fibers with boron catalytic graphitization, Compos. Sci. Technol. 200 (2020) 108455. 